\title{
Minimal model for spontaneous quantum synchronization
}

\author{
Claudia Benedetti, ${ }^{1, *}$ Fernando Galve, ${ }^{2, \dagger}$ Antonio Mandarino,,${ }^{1,3, \ddagger}$ Matteo G. A. Paris, ${ }^{1, \S}$ and Roberta Zambrini ${ }^{2, \|}$ \\ ${ }^{1}$ Quantum Technology Lab, Dipartimento di Fisica, Universitá degli Studi di Milano, I-20133 Milano, Italy \\ ${ }^{2}$ IFISC (UIB-CSIC), Instituto de Física Interdisciplinar y Sistemas Complejos (Universitat de les Illes Balears, Consejo Superior de \\ Investigaciones Científicas), Palma de Mallorca, Spain \\ ${ }^{3}$ Center for Theoretical Physics, Polish Academy of Science, 02-668 Warsaw, Poland
}

(Received 29 July 2016; published 15 November 2016)

\begin{abstract}
We show the emergence of spontaneous synchronization between a pair of detuned quantum oscillators within a harmonic network. Our model does not involve any nonlinearity, driving, or external dissipation, thus providing the simplest scenario for the occurrence of local coherent dynamics in an extended harmonic system. A sufficient condition for synchronization is established by building upon the Rayleigh normal mode approach to vibrational systems. Our results show that mechanisms favoring synchronization, even between oscillators that are not directly coupled to each other, are transient energy depletion and crosstalk. We also address the possible buildup of quantum correlations during synchronization and show that indeed entanglement may be generated in detuned systems, starting from uncorrelated states and without any direct coupling between the two oscillators.
\end{abstract}

DOI: 10.1103/PhysRevA.94.052118

\section{INTRODUCTION}

Synchronization among dynamical systems is a widespread phenomenon that has been widely studied in the classical domain and, more recently, in the quantum regime [1-4]. Indeed, synchronization is a relevant phenomenon in several contexts, including physical, biological, chemical, and social systems, and it has also been generalized to a large variety of dynamical regimes, from regular oscillations to chaotic evolutions [5-9]. These investigations have extended the definition of synchronization, showing that mutual or directional coupling between inhomogeneous components is relevant for its occurrence, as well as nonlinearity, dissipation, noise, forcing, or time delay. Overall, synchronization emerges as a paradigmatic phenomenon in complex systems [7].

The extension of the synchronization concept to the quantum case is not straightforward, since dynamical trajectories of the observables are not well defined and the very quantum properties of the canonical variables prevent the exact fulfillment of the classical conditions defining synchronization $[4,10]$. In turn, the appearance of quantum synchronization has been proved to be different from the appearance of coherence (as entanglement) and a question arises on how, whether, and when the two phenomena may coexist.

In this framework, the question about the necessary ingredients to observe spontaneous synchronization in simpler dynamical models was less explored. While in a classical setting, synchronization is mostly studied for nonlinear systems. In a recent work on the synchronization of quantum fluctuations [2], it was shown that it can actually arise even in linear models, solely due to dissipation, and it may persist asymptotically for larger systems [3,11].

\footnotetext{
*claudia.benedetti@unimi.it; users.unimi.it/aqm

†fernando@ifisc.uib-csic.es

${ }^{\ddagger}$ mandarino@cft.edu.pl

§matteo.paris@fisica.unimi.it; users.unimi.it/aqm

"roberta@ifisc.uib-csic.es
}

In this work we introduce and discuss a minimal model for the emergence of synchronization, in systems with no external forcing, nonlinear effects, and dissipation. More specifically, we consider an isolated linear network of coupled harmonic oscillators and address the emergence of coherent dynamics, i.e., synchronization, in the subsystem made of a pair of nodes. In fact, harmonic networks, besides being fundamental ingredients for modeling open quantum systems [12], are of interest for a broad spectrum of topics ranging from consensus problems [13] to trapped ions [14].

In general, harmonic network models of extended environments, even in the weak-coupling limit, lead to a rather complex dissipation mechanism for the embedded subsystems [15-18], including several different spatial effects when the system is multipartite [19,20]. Our physical model involves a large isolated network of oscillators and within this description, we analyze dissipation-induced synchronization. In addition, we provide a sufficient condition for the emergence of synchronization between detuned nodes in the framework of the physics of vibrations and in the limit of the Rayleigh approximation.

We also analyze the strong-coupling regime, where the two nodes under investigation are strongly coupled to the rest of the network. This is still a linear model amenable to analytic solution. Clearly, if one excites only one normal mode, some network nodes will oscillate synchronously, but this in not the case under study here. We consider instead a generic initial condition exciting the two probes. Here the mechanisms governing the transport of energy as well as the possible scenarios for the emergence of synchronization are far to be trivial, being typically limited to temporal transients and susceptible to variations in couplings, inhomogeneities, and boundary effects. In particular, we present and discuss two different routes to synchronization mediated by the environment in a simple chain configuration. In addition, we analyze in detail the possible buildup of quantum correlations during synchronization, showing that entanglement may be generated in our detuned systems starting from uncorrelated states and without any direct coupling between the two oscillators. 
Indeed, synchronization in itself is defined using classical temporal averages also for quantum systems. However, all the synchronization scenarios mentioned above are not specific to classical systems, since they are not limited to first-order moments. In fact, quantum noise synchronization in the presence of squeezing has already been reported [2,3], upon considering local variances. We thus address the possible emergence of quantum signatures and analyze the dynamics of quantum correlations and the possible buildup of mutual information and entanglement when starting from uncorrelated product states. The possibility to generate quantum correlations through bosonic baths has been already considered in the literature. In particular, ion chains acting as reservoirs have recently been shown to mediate entanglement between identical ions defects when placed on one edge [21] and also at a distance [22], while entanglement generation via a heat bath could not be established between remote objects in Ref. [23]. Here we take a further step, extending previous analysis to nonuniform systems (being the system components detuned), allowing for strong system-environment coupling [24], and establishing the connections with coherent (synchronous) dynamics $[2,4,25]$.

The paper is structured as follows. In Sec. II we introduce our model and establish notation. We also illustrate the quantitative measure of synchronization used throughout the paper and the Langevin equation governing the dynamics of the pair of oscillators. In Sec. III we discuss a general condition for synchronization and illustrate our results about synchronization via weak dissipation or across the chain. In Sec. IV we show the results obtained in the strong-coupling regime and discuss synchronization by coupling to a common chain mode or by crosstalk. Finally, in Sec. V we show our results about the links between synchronization and the buildup of quantum correlations for the oscillators coupled to a common chain node or to the chain edges. Section VI closes the paper with some concluding remarks.

\section{DYNAMICAL MODEL}

We consider a large network of $M$ coupled harmonic oscillators (HOs) of unit mass described by the Hamiltonian $(\hbar=1)$

$$
H_{E}=\sum_{j=1}^{M} \frac{1}{2}\left(P_{j}^{2}+\Omega_{0}^{2} X_{j}^{2}\right)+\sum_{j, k=1}^{M} A_{j k}\left(X_{j}-X_{k}\right)^{2} .
$$

In addition, we address a system of two detuned oscillators, coupled to a pair of nodes of the network with strength $K$ and coupled between them with strength $\lambda$. The dynamics of the overall system is governed by the Hamiltonian $H=$ $H_{S}+H_{E}+H_{I}$, with

$$
\begin{gathered}
H_{S}=\frac{1}{2}\left(p_{1}^{2}+p_{2}^{2}\right)+\frac{1}{2}\left(\omega_{1}^{2} x_{1}^{2}+\omega_{2}^{2} x_{2}^{2}\right)+\frac{\lambda}{2}\left(x_{1}-x_{2}\right)^{2}, \\
H_{I}=K\left(x_{1} X_{m}+x_{2} X_{n}\right),
\end{gathered}
$$

where $n, m \in[1, M]$ are the positions within the network, where the two detuned oscillators are plugged. The canonical operators for the oscillators in the chain are denoted by capital letters $\left[X_{j}, P_{k}\right]=i \delta_{j k},\left[X_{j}, X_{k}\right]=0$, and $\left[P_{j}, P_{k}\right]=0, j, k=$ $1, M$, whereas $\left[x_{j}, p_{k}\right]=i \delta_{j k},\left[x_{j}, x_{k}\right]=0$, and $\left[p_{j}, p_{k}\right]=0$, $j, k=1,2$, are the operators for the two detuned oscillators (at frequencies $\omega_{j}, j=1,2$ ). The (common) natural frequency of the oscillators in the network is denoted by $\Omega_{0}$ and the matrix $A_{j k}$ contains information about their couplings.

In the limit of $M \rightarrow \infty$ and decoupled oscillators, i.e., $\lambda=0$, this is a well-known framework for open quantum systems [12] and can be used to microscopically derive generalized Langevin equations for the reduced system dynamics [26]. In this framework, one considers a set of independent degrees of freedom in the environment, the environmental normal modes $Q_{n}$, and assumes a certain spectral density, encoding the form of the coupling between system and environment as well as the spectral distribution of the latter. However, one can go beyond phenomenological assumptions and derive the spectral density associated with more complex configurations of coupled HOs, constituting different kinds of finite networks [15-17,27]. The case of a homogeneous chain, i.e., $A_{j k}=g \delta_{|j-k|, 1}$, is particularly interesting because it allows one to (i) reproduce an Ohmic dissipation [27] and (ii) have a clear picture of the transport dynamics. On the other hand, increasing the environment complexity allows one to engineer arbitrarily complex spectral densities, as in Refs. [15-17], exhibiting non-Markovian effects [18,28].

\section{A. Synchronization}

Mutual synchronization arises when, in spite of its detuning, the pair of oscillators starts to oscillate coherently, at a common frequency. A quantitative estimation of synchronization comes from a Pearson correlation among two time-dependent functions $f$ and $g$, namely,

$$
\mathcal{C}_{f, g}(t, \Delta t)=\overline{\delta f \delta g} / \sqrt{\overline{\delta f^{2} \delta g^{2}}},
$$

where the bar stands for a time average

$$
\bar{f}=\int_{t}^{t+\Delta t} d t^{\prime} f\left(t^{\prime}\right)
$$

within a time window $\Delta t$ and $\delta f=f-\bar{f}$. This is an indicator measuring the presence of dynamical synchronization between either classical trajectories [7] or quantum systems [2] characterized by average positions, variances, and possibly higher-order moments. Other indicators of synchronization consider different forms of correlations between the nodes as in Refs. [4,25].

As recently reported [2,3], a system of two (or more) HOs weakly dissipating into an infinitely large thermal bath $(M \rightarrow$ $\infty)$ displays synchronous dynamics when one normal mode is more protected against dissipation than the other(s) [20]. In other words, synchronization emerges when all modes but one are largely damped and the dynamics is then governed by the eigenfrequency of the most robust mode [3,11]. The general condition derived for synchronization in the presence of a weakly coupled and infinite bath is indeed the presence of a gap between the damping rate of the two least damped modes of the system [3]. For a finite environment and beyond weak coupling, the scenario is more complex but richer and our first step is to identify a similar mechanism for the emergence of synchronization (see Sec. III). 


\section{B. Dynamics}

The dynamics of the subsystem of detuned oscillators, from now on referred to as the system, is governed by a pair of integro-differential equations. First- and second-order moments of the operators $\left(x_{j}, p_{j}\right), j=1,2$, are sufficient to fully characterize Gaussian states and their dynamics. We thus start by considering the average positions of the system oscillators $\left\langle x_{1,2}(t)\right\rangle$, whose dynamics is governed by generalized quantum Langevin equations [12,26]. These integro-differential equations depend on the structure and state of the overall network and for the normal modes $q_{1,2}$ of the system they read (see Appendix A)

$$
\begin{gathered}
\left\langle\ddot{q}_{1}(t)\right\rangle+\int_{0}^{t} d t^{\prime}\left[\gamma_{1}\left(t-t^{\prime}\right)\left\langle\dot{q}_{1}\left(t^{\prime}\right)\right\rangle+\eta\left(t-t^{\prime}\right)\left\langle\dot{q}_{2}\left(t^{\prime}\right)\right\rangle\right] \\
+\Lambda_{1}^{2}\left\langle q_{1}(t)\right\rangle-\gamma_{1}(0)\left\langle q_{1}(t)\right\rangle-\eta(0)\left\langle q_{2}(t)\right\rangle=0 .
\end{gathered}
$$

Analogously, an equivalent equation is found for $\left\langle q_{2}\right\rangle$ by replacing $1 \leftrightarrow 2$. Here the network features are encoded in the time-dependent coefficients $\gamma_{s}(s=1,2)$ and $\eta$, while $\Lambda_{1,2}$ are $H_{S}$ eigenfrequencies and we assume $\left\langle q_{1,2}(0)\right\rangle=0$ as initial conditions.

We emphasize that the system normal modes $q_{1,2}$ diagonalize $H_{S}$ but they remain dynamically coupled through damping, due to the interaction with the environment. In fact, the damping kernel contains different components. The first one is given by

$$
\gamma_{s}\left(t-t^{\prime}\right)=\sum_{j=1}^{M} \frac{c_{s}^{2}(j)}{\Omega_{j}^{2}} \cos \left[\Omega_{j}\left(t-t^{\prime}\right)\right] \Theta\left(t-t^{\prime}\right),
$$

with $s=1,2$, and governs the local damping at each node as well as the possible feedback from the boundaries of the finite chain. The second terms reads

$$
\eta\left(t-t^{\prime}\right)=\sum_{j=1}^{M} \frac{c_{1}(j) c_{2}(j)}{\Omega_{j}^{2}} \cos \left[\Omega_{j}\left(t-t^{\prime}\right)\right] \Theta\left(t-t^{\prime}\right)
$$

and introduces cross effects in the friction, through the transmission of signals among the system components along the chain. For this reason the $\eta$ coefficient is symmetric. The mathematical expressions for the $c_{s}(j)$ and $\Omega_{j}$ coefficients are given in Appendix A. The initial state for the network is the fundamental one $(T=0)$, being the initial energy excitation localized in the system oscillators only.

\section{A SUFFICIENT CONDITION FOR SYNCHRONIZATION}

The time nonlocal dissipation term in Eq. (4) may be approximated by a time local one, i.e., constant damping, only in specific situations [26,29]. This is usually the case when the system is weakly coupled to the rest of the network though, strictly speaking, each configuration of the network should be studied in detail to understand whether and when a time local description is appropriate, at least during a transient time. If these conditions are fulfilled, the dynamics of the system is described by a set of coupled differential equations of the form

$$
\langle\ddot{\mathbf{x}}\rangle+G\langle\dot{\mathbf{x}}\rangle+A\langle\mathbf{x}\rangle=0
$$

where $\mathbf{x}=\mathbf{x}_{1}, \mathbf{x}_{2}$ and $A$ and $G$ are time-independent matrices. An interesting question, addressed earlier by Lord Rayleigh [30] in the context of the vibration of structures [30,31], is whether normal modes may be individuated in spite of the presence of dissipation. The undamped dynamics follows from a superposition of normal modes obtained by diagonalizing the stiffness matrix $G$ and the coupling one $A$ in Eq. (6), but the specific form of damping undermines this description because, in general, $A$ and $G$ cannot be simultaneously diagonalized.

As a matter of fact [30,32], classical normal modes [33] are present if the matrices $A$ and $G$ commute. This leads to a simple description for the independently damped normal modes of the free dynamics $\left(\mathbf{q}=\left(q_{1}, q_{2}\right)\right)$. Small deviations from the condition $[G, A]=0$ justify Rayleigh's approximation of neglecting the nondiagonal components of $G$ in the basis of $\mathbf{q}$. This corresponds to the so-called reduction method [30] of disregarding off-diagonal terms of $G^{\prime}=M^{-1} G M$, where $M=$ $\left(\left\{q_{1}\right\},\left\{q_{2}\right\}\right)$ diagonalizes $A\left(A^{\prime}=M^{-1} A M\right)$, which is useful when dissipation is small. The approximation is equivalent to neglecting the small cross damping among natural vibrations and of course the validity of this approach depends on the relative size when comparing with self-dampings. An example is shown in Ref. [34] and is based on a secular approximation. The model described in Eq. (6), simplified under Rayleigh's approximation, allows for a necessary and sufficient condition for synchronization: A pair of detuned oscillators embedded in a network will synchronize if there exists a gap between the normal mode damping rates $G_{11}^{\prime}$ and $G_{22}^{\prime}$. This condition is general for dissipation in infinite baths [3], while for finite systems it is limited to the transient where the average dynamics of the pair of oscillators can be approximated by Eq. (6). Significant buildup of synchronization requires the least damped mode to be suppressed and this phenomenon should occur in a time scale of the order of the inverse of the larger damping

$$
\tau_{S}^{-1} \sim \max \left(\left|G_{11}^{\prime}\right|,\left|G_{22}^{\prime}\right|\right)
$$

In the following section we consider a finite chain configuration and provide an example of the application of the above condition.

\section{A. Synchronization via weak dissipation}

Let us now consider a network made of a chain of $M$ oscillators homogeneous in frequency and couplings. The Hamiltonian is given by

$$
H_{E}=\sum_{j=0}^{M} \frac{p_{j}^{2}}{2}+\frac{1}{2} \Omega_{0}^{2} X_{j}^{2}+\frac{g}{2}\left(X_{j}-X_{j+1}\right)^{2} .
$$

The network acts as an environment for a system made of two oscillators attached at one edge of the chain. The interaction Hamiltonian reads

$$
H_{I}=K\left(x_{1}+x_{2}\right) X_{1}
$$

In this configuration only $x_{+}=x_{1}+x_{2}$ is directly coupled to the chain and $x_{ \pm}$diagonalize the damping term, while the system Hamiltonian $H_{S}$ is diagonal in $q_{1,2}$. 

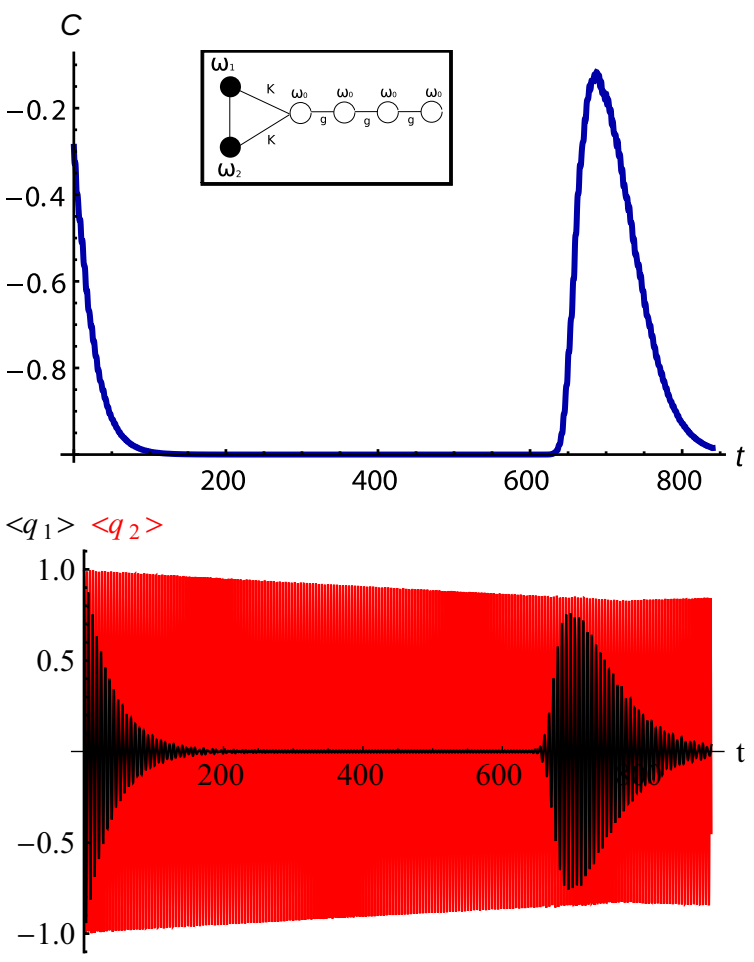

FIG. 1. Synchronization measure $C(t)$ and dynamics for $\omega_{2} / \omega_{1}=1.1, \lambda=0.5 \omega_{1}^{2}$, and $x_{1}(0)=0.14 \omega_{1}^{-1 / 2}$. The other parameters are $x_{2}(0)=1.4 \omega_{1}{ }^{-1 / 2}, M=300, \Omega_{0}=0.4 \omega_{1}, g=1.2 \omega_{1}^{2}$, and $K=0.2 \omega_{1}^{2}$. In the lower panel the black line denotes the function $\left\langle q_{1}(t)\right\rangle$ and the red one is for $\left\langle q_{2}(t)\right\rangle$. Here $\left\langle q_{1,2}\right\rangle$ are in units of $\omega_{1}^{-1 / 2}$ and time is in units of $\omega_{1}^{-1}$.

In the limit of an infinitely large chain and vanishing local potential $\left(\Omega_{0} \simeq 0\right)$ the environment acts as an Ohmic bath and the ratio between the damping rates is

$$
G_{11}^{\prime} / G_{22}^{\prime} \simeq \frac{1+\sin 2 \theta}{1-\sin 2 \theta},
$$

where the parameter $\theta$ depends upon the detuning and the coupling $\lambda$ (see Appendix A). The sufficient condition for transient synchronization is the presence of a gap between the normal modes damping, as it happens for small detuning, i.e., $\left|\omega_{1}-\omega_{2}\right|^{2} / \lambda<1$ in the case of Fig. 1 , where $\sin 2 \theta \simeq 1$. The synchronization measure $\mathcal{C}$ in this case shows that perfect antisynchronization is present up to the revival time $\tau_{R} \sim$ $2 M / \omega_{1}$. If the coupling of one of the oscillators to the chain switches from attractive to repulsive, $K x_{2} X_{1} \rightarrow-K x_{2} X_{1}$ in Eq. (8), then the quantity $x_{-}=x_{1}-x_{2}$ couples to the chain and synchronization instead of antisynchronization arises.

As a matter of fact, during the initial transient time finite-size effects can be neglected and the energy of the two system's oscillators flows into the environmental chain [35], leading to an effective dissipation into a common bath [2,3]. Therefore, the neat buildup of antisynchronization of Fig. 1 is consistent with the predicted phenomenon of Ref. [2], where an infinite bath was considered. Boundary effects cause a departure from the Ohmic dissipation [constant damping in Eq. (4)], leading to revivals. Figure 1 shows that reflection from the boundary at $t=\tau_{R}$ actually deteriorates the coherent dynamics between the pair of HOs and similar results are found when there are defects in the chain causing feedback effects at shorter times. This is accompanied by a regrowth in the oscillation amplitude of the damped mode $q_{1}$. The loss of antisynchronization is indeed due to a common forcing toward synchronization due to the feedback signal reflected at the edge of the chain. At later times $\left(t>\tau_{R}\right)$, after a competition transient, antisynchronization is restored under the effect of dissipation, as shown in Fig. 1, lasting until feedback effects arise again at $t \sim 2 \tau_{R}$.

\section{B. Synchronization across the chain}

A natural question is what happens when moving the second oscillator through the chain with system-environment interaction

$$
H_{I}=K x_{1} X_{1}+K x_{2} X_{m}, \quad m \in[1, M] .
$$

The dependence of dissipation on the distance $m$ in the weak-coupling regime has been described elsewhere [20] for an infinite environment and a periodic transition between dissipation in common and separate baths has been predicted. The case under study differs due to finite-size effects: Reflections from the boundaries and crosstalk between the oscillators and feedback signals lead to a dynamics that strongly depends on the plugging distance $m$ and perfect synchronization may arise or not just by moving the system components from one site to the neighboring one. Still, this sensitivity to the plugging position is absent during a transient when $0 \ll m \ll N$, i.e., second oscillator far from the first one and from the edge of the chain. More details are given in Appendix B.

\section{SYNCHRONIZATION IN THE STRONG-COUPLING REGIME}

The mechanism of synchronization by dissipation is enabled by the presence of coupling between the system oscillators (i.e., $\lambda \neq 0$ ) and it is consistent with results obtained for infinite environments [2,3]. An interesting question is the possibility to synchronize detuned oscillators in the absence of a direct coupling between them, i.e., $\lambda=0$, solely due to the mediating effect of the rest of the network. This was actually shown to be possible for spins in Ref. [36], but it does not occur for weakly coupled harmonic oscillators. Indeed, for the oscillator pair attached to a common node, the dissipation mechanism described above does not produce synchronization in the weak-coupling regime. Inspection of the master equation in [2] shows that, even for long chains (large $\tau_{R}$ ), the effective coupling induced by the bath (Lamb shift) is actually too small to lead to significant synchronization before the system thermalizes. On the other hand, a full system-bath model allows one to address less explored strong dissipation regimes, enabling dynamical scenarios for synchronization that are not present for weak coupling.

\section{A. Coupling to a common chain node}

We now consider a configuration where the system is attached to one edge of the environment chain, as in Eq. (8), but now the two oscillators are uncoupled, $\lambda=0$. We allow for a frequency detuning $\omega_{1} \neq \omega_{2}$, implying that $x_{ \pm}$are not the eigenmodes of $H_{S}$. Up to the revival time $\tau_{R}$, the system 


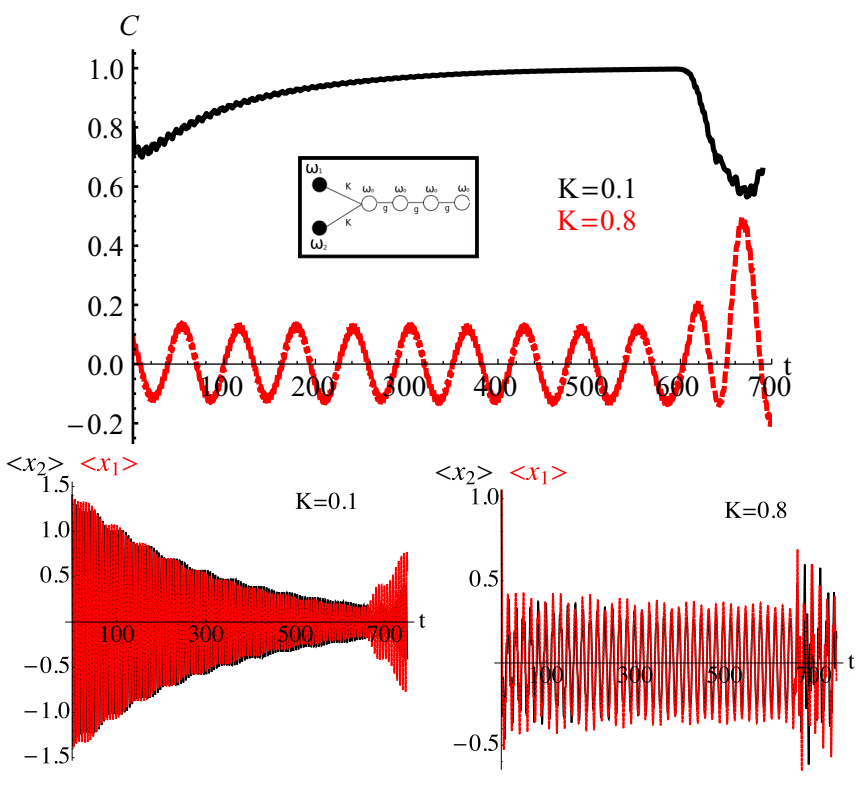

FIG. 2. Shown in the top plot is the synchronization measure $C(t)$ as a function of time for two different values of the coupling $K=0.1$ (solid black) and 0.8 (dotted red), in units of $\omega_{1}^{2}$. The other parameters are fixed: $\omega_{2} / \omega_{1}=1.1, \lambda=0, M=300, \Omega_{0}=0.4 \omega_{1}$, and $g=1.2 \omega_{1}^{2}$. In the inset we show a schematic representation of the considered configuration. The bottom plot shows the dynamics of the two oscillators plugged into the first node of the chain, with $K=0.1$ (left) and $K=0.8$ (right), in units of $\omega_{1}^{2}$. Here $\left\langle x_{1,2}\right\rangle$ are in units of $\omega_{1}^{-1 / 2}$ and time in units of $\omega_{1}^{-1}$.

oscillators will dissipate into the common environment and no synchronization is possible for weak coupling, i.e., $K \ll \omega_{i}^{2}$. Underdamped detuned oscillations characterize the dynamics at all times and the system components $x_{1}$ and $x_{2}$ remain incoherent.

This is not the case when $K \lesssim \omega_{i}^{2}$. We first notice that this rather large dissipation does not completely deplete the system energy. Indeed, after a fast transient oscillatory decay, the system achieves a steady regime of rather large oscillations with constant amplitude that last up to the revival time. For the choice of parameters of Fig. $2, \tau_{R} \simeq 600 / \omega_{1}$. In this regime, oscillations are coherent at a frequency smaller than the system frequencies $\left(0.4<\omega_{1,2}\right.$ in Fig. 2 , bottom right $)$ and perfect synchronization emerges. After the transmission of the initial pulse, originated at the edge of the chain, the stiff coupling ( $K=0.8 \omega_{1}^{2}$ in Fig. 2 ) to the environment leads to a steady state in which the system vibrates at the lowest frequency of the chain $\Omega_{0}=0.4 \omega_{1}$. For decreasing coupling the system depletion of energy continues until complete damping, whereas for weaker coupling (e.g., $K=0.1 \omega_{1}^{2}$ ) the system shows underdamped oscillation at the detuned (Lamb shifted) natural detuned frequencies, so no synchronization is established. This scenario of synchronization occurs for uncoupled probes stiffly attached to one node of a network until signal reflections (depending on the network topology) drive the system away from coherent oscillation, as shown here at the revival time.

\section{B. Synchronization by crosstalk}

The phenomena described in Secs. III A and IV A show how boundary effects are often detrimental for synchronization. A different dynamics, however, may take place if the two oscillators are allowed to exchange their energy across the system. To illustrate this effect we consider a configuration where the oscillators do not interact directly, i.e., $\lambda=0$, and are plugged at the opposite edges of a chain (see Fig. 3). During an initial transient, even if the probes are attached to the same environment (the chain), there are no decoherence-free subspaces [20]: The probes actually experience independent dissipation, the $\eta$ kernel vanishes, and $x_{+}$and $x_{-}$are coupled to orthogonal modes of the chain. During this transient the system oscillators lose energy and do not synchronize, as shown in Figs. 3(a) and 3(b). This is consistent with previous studies with infinite and separate environments [2].

In the weak-coupling regime, the undamped oscillators start to experience the effect of each other after the time interval needed for signal propagation through the system, but their dynamics still remain incoherent. On the other hand, for stronger dissipation $K \lesssim \omega_{1,2}^{2}$ a sudden rise of the system oscillations appears at the crosstalk time $\tau_{C T}=\tau_{R} / 2$ and perfect synchronization emerges. This behavior is illustrated in Fig. 3, in the central regions of Figs. 3(a) and 3(b). For $t>\tau_{R}$ each oscillator starts to receive its own feedback and synchronization is lost again, since it is driven back to its natural frequency.

The mechanism of synchronization found in this regime consists of a reciprocal driving force of the two-system HOs after their local damping: At $\tau_{C T}$ they have lost their initial energy due to their dissipation into the cold chain $\left(K=0.2 \omega_{1}^{2}\right.$ in Fig. 3) and in the crosstalk time window $\tau_{C T}<t<\tau_{R}$ they receive a signal from the opposite (detuned) system oscillator. A driving at the frequency of the opposite oscillator, being detuned, would not cause any synchronization, but actually the exchanged signals are not at a single frequency, having a broad bandwidth due to the transmission through the chain. We find that, within the crosstalk time window, the oscillator 1 is driven by a signal containing both the main frequency component $\omega_{2}^{\prime}$ [37] and the resonant one $\omega_{1}^{\prime}$ (present in the broad signal transmitted through the chain), leading to the beating signal observed in Fig. 3(c). A similar scenario occurs for the other oscillator 2, now with the strongest and resonant frequency component exchanged. Therefore, the oscillators placed at the edges, during crosstalk time, experience a driving force at a signal with the two detuned frequencies, leading to the characteristic beating signal of Fig. 3 and to perfect synchronization.

This mechanism of synchronization for crosstalk is based on a reciprocal effect between the system components and is robust when breaking the symmetry in the initial conditions, even though in this case delayed synchronization arises. Indeed, for nonidentical initial states $\left\{x_{1}(0), p_{1}(0)\right\}$ and $\left\{x_{2}(0), p_{2}(0)\right\}$, the respective signals experience a relative phase delay. Time delay can be taken into account by considering the delayed signals $\left\langle x_{1}(t)\right\rangle$ and $\left\langle x_{2}(t+\Delta t)\right\rangle$ in $C$. Since this synchronization scenario is very sensitive to the initial conditions, both antisynchronization and synchronization may arise. 
(a)

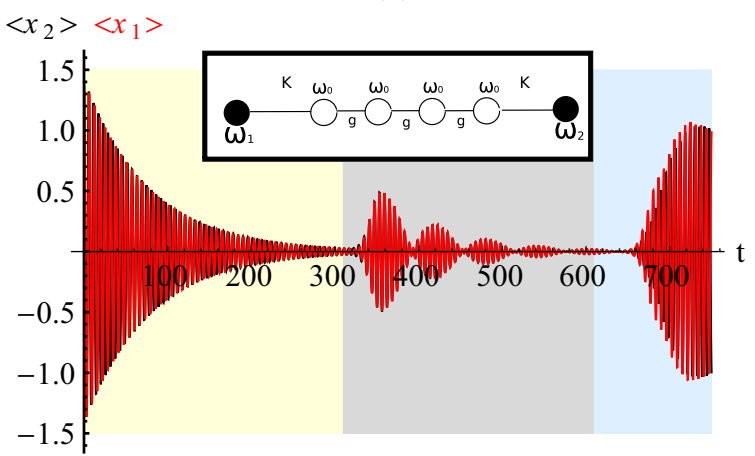

(b)

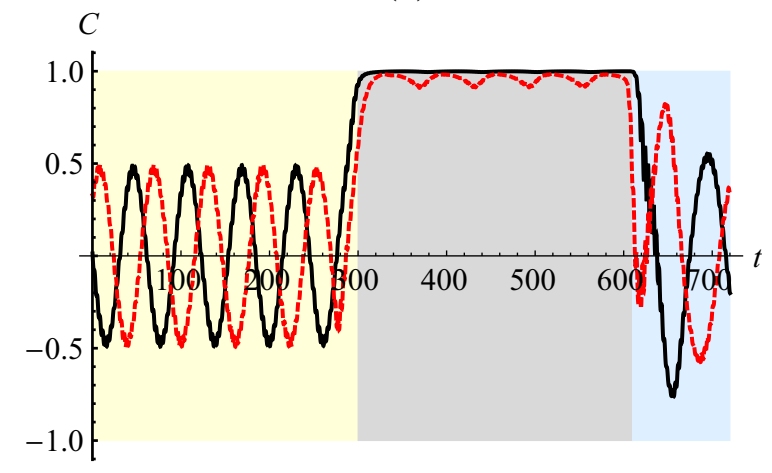

(c)

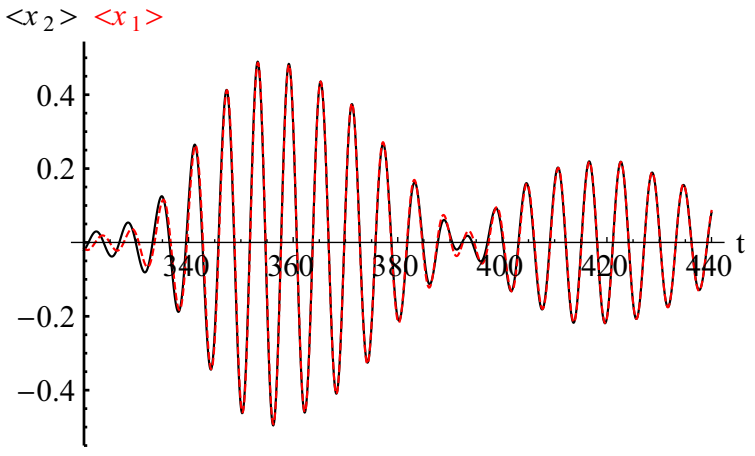

FIG. 3. (a) Dynamics, (b) synchronization measure $C(t)$, and (c) enlarged view of the dynamics for two uncoupled oscillators $(\lambda=0)$, with detuning $\delta \omega / \omega_{1}=0.1$ coupled to the extremities of the oscillator chain with coupling constant $K=0.2 \omega_{1}^{2}$ and $M=300, \Omega_{0}=0.4 \omega_{1}$, and $g=1.2 \omega_{1}^{2}$. The inset in (a) shows a schematic representation of the configuration under analysis. (b) Synchronization measure for two different initial conditions: symmetric $x_{1}(0)=x_{2}(0)=1.4 \omega_{1}^{-1 / 2}$ and $p_{1}(0)=p_{2}(0)=0$ (solid black line) and asymmetric $x_{1}(0)=$ $x_{2}(0)=2 \omega_{1}^{-1 / 2}$ and $p_{1}(0)=0,=p_{2}(0)=10 \sqrt{\omega_{1}}$ (dashed red line). (c) Dynamics of the two oscillators $\left\langle x_{1}(t)\right\rangle$ (solid black line) and $\left\langle x_{2}(t)\right\rangle$ (dashed red line) for a time window where synchronization appears. The background colors in (a) and (b) are inserted as a guide for the eye to mark the three regions of independent dissipation (pink), crosstalk (blue), and revival (light blue). Here $\left\langle x_{1,2}\right\rangle$ are in units of $\omega_{1}^{-1 / 2}$ and time is in units of $\omega_{1}^{-1}$.

\section{SYNCHRONIZATION AND QUANTUM CORRELATIONS}

An interesting question to answer is whether the emergence of synchronization is accompanied by an increase in the quantum or classical correlations in the system and if the mechanism of synchronization by dissipation (Sec. III A) may be evidence for the appearance of robust quantum correlations and entanglement between the oscillators pair. As reported in [2], starting from an entangled state weakly dissipating into the environment, decoherence and deterioration of quantum correlations will be reduced in the presence of synchronization. This mechanism has been analyzed also for three oscillators [11] and in networks [3].

Here we consider instead the possibility to create correlations and entanglement starting from product states of the system oscillators and in relation to quantum synchronization. To this purpose we consider uncoupled system oscillators starting from an uncorrelated (product) state with local squeezing. For identical oscillators $\omega_{1}=\omega_{2}$, entanglement mediated by the reservoir chain and its dynamical (sudden-death and revival) features have been predicted in Refs. [21,22,38] in symmetric models. The possibility to entangle two oscillators due to strong dissipation in a common bath was addressed in [24], while in [3] the case of a dissipative network was treated. The issue we are interested in here is the possibility to create entanglement due to the coherent energy transmission across the environment between detuned oscillators and in relation to spontaneous synchronization. The cases of interest are for system coupling mediated by the chain $(\lambda=0)$. We monitor the system entanglement given by the logarithmic negativity $\mathcal{E}=\max (0,-\ln v)$, with $v$ the smallest symplectic eigenvalue of the partially transposed density matrix [39]. Further, we consider the mutual information $\mathcal{M}=S_{A}+S_{B}-S_{A B}$, with $S_{i}$ the von Neumann entropy of the reduced system $i=1,2$ and $S_{A B}$ the total entropy. Actually, the latter has also been suggested to be an order parameter for quantum synchronization [25].

\section{A. Coupling to a common chain node}

For a system plugged at the same point of a chain we have seen that two otherwise uncoupled oscillators $(\lambda=0)$ can synchronize in the strong-coupling regime due to the mediating effect of the environment (see Sec. IV A). We find that this synchronization scenario is also present for system oscillators in vacuum squeezed states. In this case synchronization arises between the second-order moments, as shown in Fig. 4. As for the case of average positions, fluctuations synchronization is allowed by the strong dissipation [Fig. 4(a)] and it later (at $\tau_{R}$ ) deteriorates due to feedback effects. Initially, both mutual information and entanglement are established between the decoupled and initially uncorrelated system oscillators, as expected, due to their strong coupling mediated by the chain and the initial local squeezing [Fig. 4(b)]. After a transient oscillatory decay $\mathcal{E}$ and $\mathcal{M}$ both reach a steady nonvanishing value, consistently with predictions in Ref. [24]. Here also synchronization appears, as shown in Fig. 4(c), and actually witnesses entanglement.

This microscopic model shows that strong coupling to a common environment allows one to synchronize and entangle uncoupled detuned oscillators whose interaction is mediated by the environment. This would persist for an infinite bath, while feedback effects in a finite model case hinder synchronization [see Fig. 4(c) for $t>\tau_{R}$ ]. Further, we notice that the increase of mutual information at $\tau_{R}$ does not always reflect 


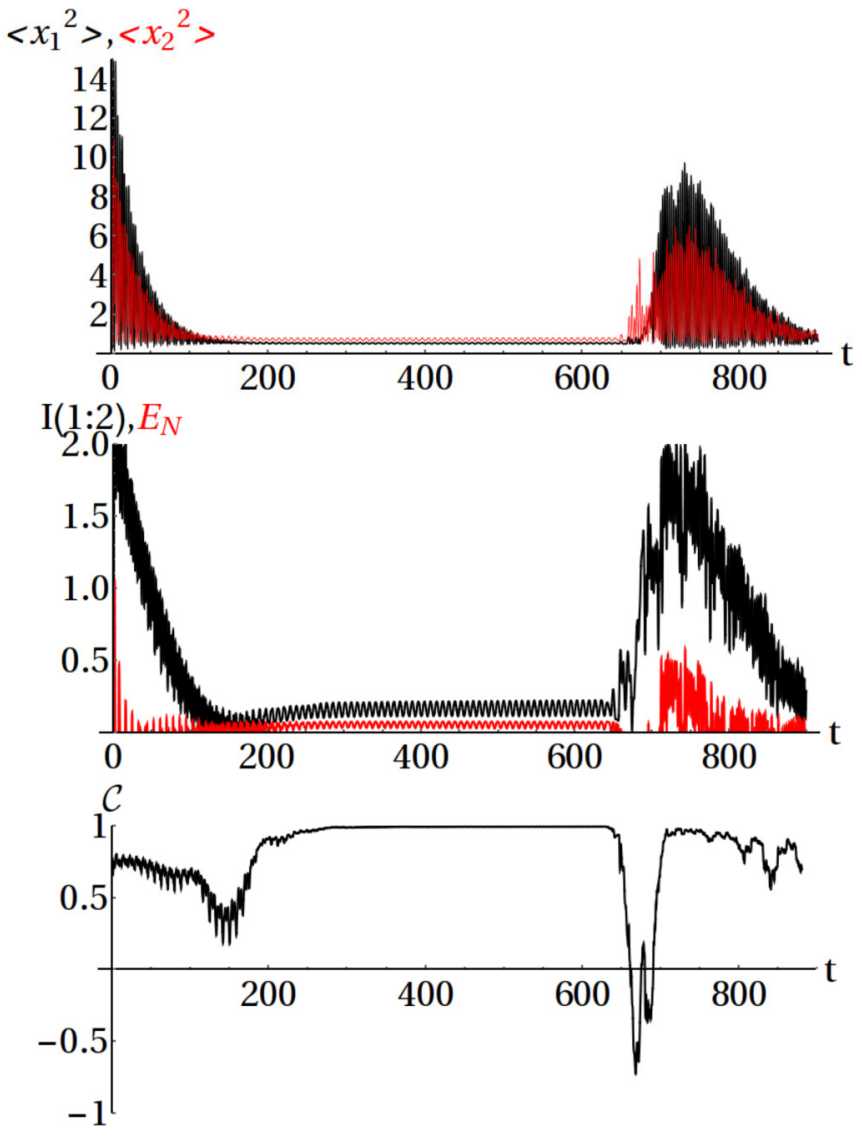

FIG. 4. Shown from top to bottom are quadratures, mutual information and entanglement, and the synchronization factor, starting from a separable squeezed state with squeezing parameters $r_{1}=r_{2}=2$, frequencies $\omega_{2} / \omega_{1}=1.2$, and coupling $\lambda=0$. The two oscillators are strongly coupled to the reservoir $K=0.8 \omega_{1}^{2}$ and the bath parameters are the same as in previous figures. Here $\left\langle x_{1,2}^{2}\right\rangle$ are in units of $\omega_{1}^{-1}$ and time is in units of $\omega_{1}^{-1}$.

dynamical synchronization that, at the contrary, can decay $\left(\mathcal{C} \simeq 0\right.$ for $\left.650<\omega_{1} t<700\right)$. The fact that two uncoupled oscillators, interacting with a chain, evolve into a synchronized and entangled state is a distinctive effect of strong coupling, which is not present for weak coupling.

\section{B. Coupling to the chain edges}

We now consider the case in which the oscillators are far apart, at the opposite edges of the chain as in Sec. IV B. Is it possible to synchronize their quantum fluctuations and entangle them due to the crosstalk? We consider again squeezed vacuum states, observing that the system probes at the opposite edges of the chain evolve toward a quantum synchronized state in its fluctuations, with a buildup of correlations during the crosstalk time, as shown by their mutual information rising from vanishing to finite values (Fig. 5). Nevertheless, for reasonable values of the initial squeezing, entanglement is never created, independently of the initial squeezing strength. For distant probes, therefore, synchronization may emerge when in a crosstalk regime where they exchange energy, but this does not lead to entanglement.
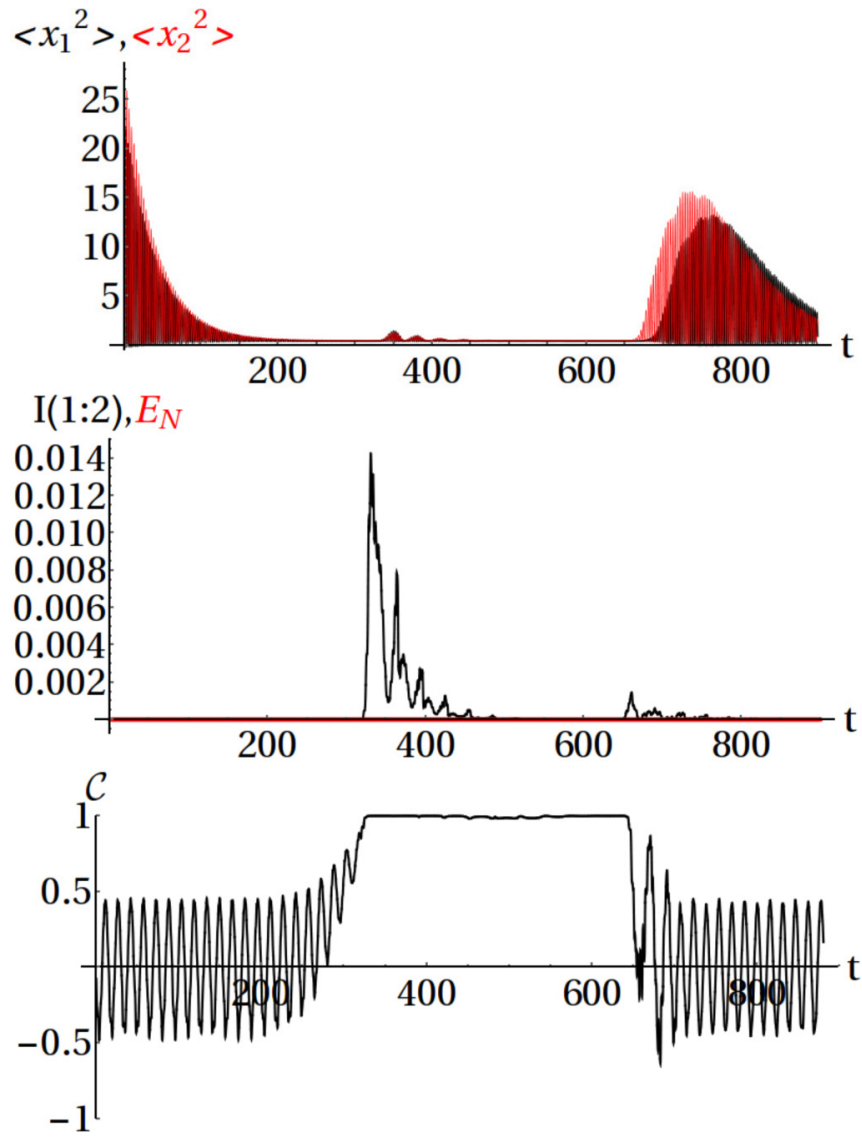

FIG. 5. Show from top to bottom are the position variances, mutual information and entanglement, and synchronization factor, starting from a separable squeezed state with squeezing parameters $r_{1}=r_{2}=2$, frequencies $\omega_{2} / \omega_{1}=1.2$, and coupling $\lambda=0$. The coupling to the reservoir is $K=0.2 \omega_{1}^{2}$ and the bath parameters are the same as in previous figures. Here $\left\langle x_{1,2}^{2}\right\rangle$ are in units of $\omega_{1}^{-1}$ and time is in units of $\omega_{1}^{-1}$.

\section{CONCLUSION}

We have addressed synchronization of two quantum oscillators within a finite linear system and analyzed in detail the possible mechanisms leading to a coherent dynamics of the (detuned) system components. In addition, we have analyzed in detail the connections of synchronization with the buildup of entanglement, starting from uncorrelated states and without any direct coupling between the two oscillators.

Our microscopic description has allowed us to go beyond the weak dissipation limit, showing that in the strong-coupling regime synchronization mechanisms appears among uncoupled oscillators, leading to coherent dynamics enabled by the environment. Furthermore, crosstalk effects may have a constructive role, inducing synchronization mediated by signal transmission. More in general, a condition for spontaneous synchronization of linear oscillators has been discussed in the context of the Rayleigh model for vibrations physics.

As a matter of fact, synchronization is important in different contexts but is not always desirable. For quantum networks [3], quantum synchronization witnesses the presence of quantum correlations, which are more robust against dissipation, and 
even the appearance of noiseless subsystems. On the other hand, in the context of the physics of vibrations, the fact that some vibration modes are damped out very slowly may compromise the stability of complex structures [31].

Our results pave the way for the analysis of local synchronization mechanisms for small clusters within a larger network and for applications of interest for quantum technology and metrology, e.g., the use of spontaneous synchronization to witness quantum correlations or the synchronization of clocks by coherent coupling.

\section{ACKNOWLEDGMENTS}

This work was supported by EU through the Collaborative Project QuProCS (Grant Agreement No. 641277) and by the Erasmus LLP placement program, by MINECO (Grant No. FIS2014-60343-P), by the "Vicerectorat d'Investigació i Postgrau" of the UIB, and by the UIB visiting professors program.

\section{APPENDIX A: HAMILTONIAN NORMAL MODES AND THE LANGEVIN EQUATION}

Let us consider a system of two quantum harmonic oscillators, characterized by frequencies $\omega_{1}$ and $\omega_{2}$ and coupling strength $\lambda$ between them. The oscillators are plugged with strength $K$ into a homogeneous chain of quantum HOs at frequency $\Omega_{0}$ and chain stiffness $g$. We now introduce the notation used to describe the system and environment normal modes (NMs)

$$
\begin{gathered}
H_{S}=\frac{{\tilde{p_{1}}}^{2}}{2}+\frac{{\tilde{p_{2}}}^{2}}{2}+\frac{1}{2} \Lambda_{-}^{2} q_{1}^{2}+\frac{1}{2} \Lambda_{+}^{2} q_{2}^{2}, \\
H_{E}=\sum_{j=1}^{M}\left[\frac{\tilde{P}_{j}^{2}}{2}+\frac{1}{2} \Omega_{j}^{2} Q_{j}^{2}\right], \\
H_{I}=q_{1} \sum_{j=1}^{M} c_{1}(j) Q_{j}+q_{2} \sum_{j=1}^{M} c_{2}(j) Q_{j},
\end{gathered}
$$

where the position normal mode operators are denoted by $q_{1,2}$ for the system and $Q_{j}$ for the environment and are computed as

$$
\begin{gathered}
q_{1}=\cos (\theta) x_{1}+\sin (\theta) x_{2}, \\
q_{2}=-\sin (\theta) x_{1}+\cos (\theta) x_{2}, \\
X_{j}=\sqrt{\frac{2}{M+1} \sum_{k=0}^{M} \sin \left(\frac{\pi k j}{M+1}\right) Q_{k},}
\end{gathered}
$$

where the quantity $\theta$ is defined by the relation

$$
\tan (2 \theta)=\frac{2 \lambda}{\omega_{2}^{2}-\omega_{1}^{2}}
$$

The eigenfrequencies and the coupling coefficients are given by

$$
\Lambda_{s}^{2}=\lambda+\frac{\omega_{1}^{2}+\omega_{2}^{2}}{2}+\frac{(-1)^{s}}{2} \sqrt{4 \lambda^{2}+\left(\omega_{1}^{2}-\omega_{2}^{2}\right)^{2}},
$$

$$
\begin{aligned}
\Omega_{j}^{2}= & \Omega_{0}^{2}+4 g \sin ^{2}\left(\frac{\pi j}{2(M+1)}\right), \\
c_{1}(j)= & \sqrt{\frac{2 K^{2}}{M+1}} \\
& \times\left[\cos \theta \sin \left(\frac{\pi j m}{M+1}\right)+\sin \theta \sin \left(\frac{\pi j n}{M+1}\right)\right], \\
c_{2}(j)= & \sqrt{\frac{2 K^{2}}{M+1}} \\
& \times\left[\cos \theta \sin \left(\frac{\pi j n}{M+1}\right)-\sin \theta \sin \left(\frac{\pi j m}{M+1}\right)\right] .
\end{aligned}
$$

The dynamics of the system is described by generalized quantum Langevin equations (GQLEs) for operators $q_{1}(t)$ and $q_{2}(t)$, obtained by starting from the set of Heisenberg equations for system and environment operators $\left\{q_{1(2)}, \tilde{p}_{1(2)}, Q_{j}, \tilde{P}_{j}\right\}$. The GQLEs are integro-differential equations that describe the dynamics of the NM operators as a function of the environment parameters and coupling constants

$$
\begin{aligned}
\ddot{q}_{1}(t) & +\left[\Lambda_{1}^{2}-\gamma_{1}(0)\right] q_{1}(t) \\
& +\int_{0}^{t} d t^{\prime}\left[\gamma_{1}\left(t-t^{\prime}\right) \dot{q}_{1}\left(t^{\prime}\right)+\eta\left(t-t^{\prime}\right) \dot{q}_{2}\left(t^{\prime}\right)\right] \\
= & -\xi_{1}(t)-\gamma_{1}(t) q_{1}(0)-\eta(t) q_{2}(0)+\eta(0) q_{2}(t)
\end{aligned}
$$

and an equivalent expression is found for operator $q_{2}$ by replacing $1 \leftrightarrow 2$. The kernels $\gamma$ and $\eta$ are expressed as

$$
\begin{gathered}
\gamma_{s}\left(t-t^{\prime}\right)=\sum_{j=1}^{M} \frac{c_{s}^{2}(j)}{\Omega_{j}^{2}} \cos \left[\Omega_{j}\left(t-t^{\prime}\right)\right] \Theta\left(t-t^{\prime}\right), \\
\eta\left(t-t^{\prime}\right)=\sum_{j=1}^{M} \frac{c_{1}(j) c_{2}(j)}{\Omega_{j}^{2}} \cos \left[\Omega_{j}\left(t-t^{\prime}\right)\right] \Theta\left(t-t^{\prime}\right),
\end{gathered}
$$

where $s=1,2$ and the external force operator $\xi_{1(2)}(t)$ depends upon the environment initial conditions

$$
\xi_{s}(t)=\sum_{j} c_{s}(j)\left(Q_{j}(0) \cos \left(\Omega_{j} t\right)+\frac{P_{j}(0)}{\Omega_{j}} \sin \left(\Omega_{j} t\right)\right)
$$

and gives a zero contribution when averaged over the vacuum state of the environment.

\section{APPENDIX B: SYNCHRONIZATION ACROSS THE CHAIN: CASE $0 \ll m \ll N$}

When moving the second oscillator through the chain but far from the edges, i.e., $0 \ll m \ll N$, there is an initial time transient in which the oscillators do not synchronize and this behavior is independent of the position $m$, as shown in Fig. 6. This occurs only before crosstalk and feedback from the boundaries take place and actually corresponds to a good approximation of independent dissipation of the two detuned systems. In particular, if the coupling is weak and time is 


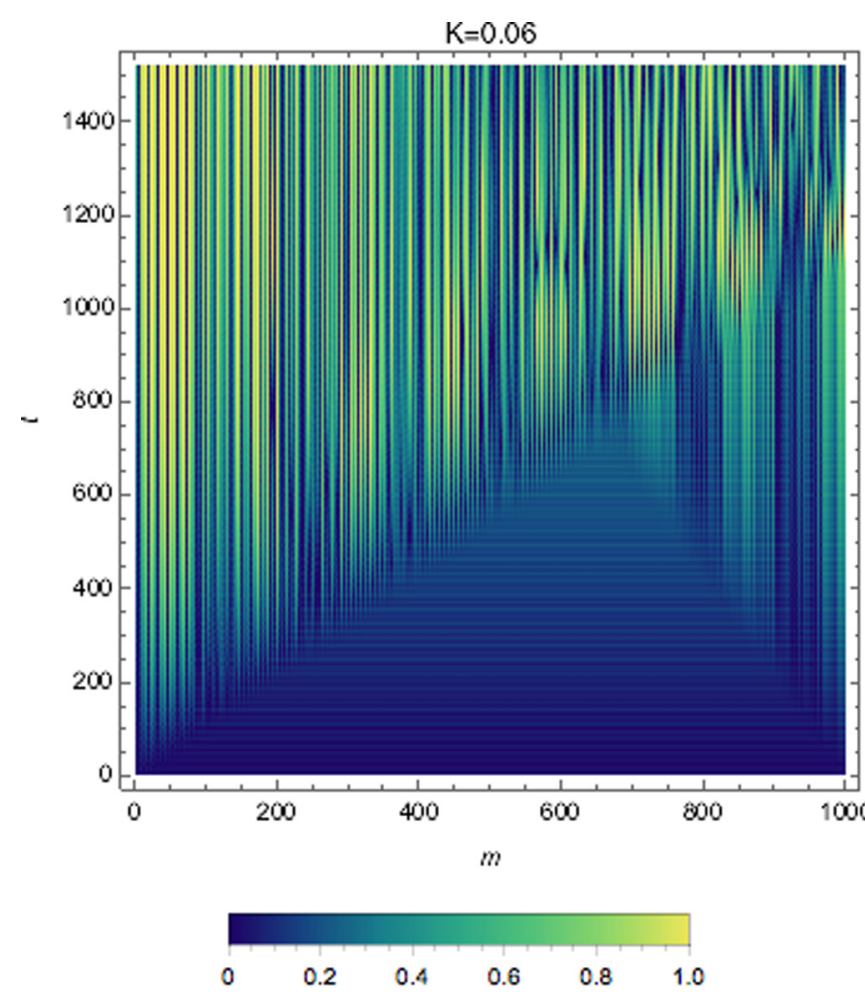

FIG. 6. Density plot of the synchronization measure $C(t)$ for a 300 -site chain as a function of the plugging site $m$ and the time $t$. The parameters are set as $\omega_{2} / \omega_{1}=1.1, \lambda=0.5 \omega_{1}^{2}, K=0.06 \omega_{1}^{2}$, $\Omega_{0}=0.4 \omega_{1}$, and $g=1.2 \omega_{1}^{2}$. long enough to have only the resonant system-bath interaction surviving, then one expects that the chain normal modes $Q_{k} \pm$ that are resonant with the system eigenfrequencies dominate the dynamics $\left(k_{-}\right.$resonates with the system normal mode $q_{1}$ and $k_{+}$with $q_{2}$ ), leading to an effective interaction

$$
H_{I}=K \sqrt{\frac{2}{M+1}}\left[c_{1} q_{1} Q_{k_{-}}+c_{2} q_{2} Q_{k_{+}}\right],
$$

with

$$
\begin{aligned}
& c_{1}=\left[\cos \theta \sin \left(\frac{\pi k_{-}}{M+1}\right)+\sin \theta \sin \left(\frac{\pi k_{-} m}{M+1}\right)\right], \\
& c_{2}=\left[\cos \theta \sin \left(\frac{\pi k_{+} m}{M+1}\right)-\sin \theta \sin \left(\frac{\pi k_{+}}{M+1}\right)\right] .
\end{aligned}
$$

Nevertheless, such an effective resonant interaction is established after long times, while during the transient analyzed here there are bands of normal modes that are exchanging energy with the system. The average effect of several such modes of the chain leads to $c_{1} \sim \cos \theta \sin \left(\frac{\pi k_{-}}{M+1}\right)$ and $c_{2} \sim$ $\sin \theta \sin \left(\frac{\pi k_{+}}{M+1}\right)$, so the system normal modes decay at some rate independently of their distance $m$, as shown in the triangular region in Fig. 6. The spatiotemporal synchronization diagram shown in Fig. 6 clearly displays the effects of crosstalk and reflections from the boundaries, leading to a strong and nonmonotonic dependence on $m$ and often to synchronization for larger times.
[1] G. Heinrich, M. Ludwig, J. Qian, B. Kubala, and F. Marquardt, Phys. Rev. Lett. 107, 043603 (2011); M. Ludwig and F. Marquardt, ibid. 111, 073603 (2013).

[2] G. L. Giorgi, F. Galve, G. Manzano, P. Colet, and R. Zambrini, Phys. Rev. A 85, 052101 (2012).

[3] G. Manzano, F. Galve, G. L. Giorgi, E. Hernandez-Garcia, and R. Zambrini, Sci. Rep. 3, 1439 (2013).

[4] A. Mari, A. Farace, N. Didier, V. Giovannetti, and R. Fazio, Phys. Rev. Lett. 111, 103605 (2013).

[5] S. H. Strogatz and I. Stewart, Sci. Am. 269, 102 (1993).

[6] G. V. Osipov, J. Kurths, and C. Zhou, Synchronization in Oscillatory Networks (Springer, Berlin, 2007).

[7] A. Pikovsky, M. Rosenblum, and J. Kurths, Synchronization: A Universal Concept in Nonlinear Sciences (Cambridge University Press, Cambridge, 2001).

[8] S. H. Strogatz, Nonlinear Dynamics and Chaos: With Applications to Physics, Biology, Chemistry, and Engineering (Westview, Boulder, 2001).

[9] S. C. Manrubia, A. S. Mikhailov, and D. H. Zanette, Emergence of Dynamical Order: Synchronization Phenomena in Complex Systems, Lecture Notes in Complex Systems Vol. 2 (World Scientific, Singapore, 2004).

[10] F. Galve, G. L. Giorgi, and R. Zambrini, Lectures on General Quantum Correlations and their Applications, edited by F. Fanchini, D. Soares-Pinto, and G. Adesso (Springer, Berlin, 2017).
[11] G. Manzano, F. Galve, and R. Zambrini, Phys. Rev. A 87, 032114 (2013).

[12] U. Weiss, Quantum Dissipative Systems (World Scientific, Singapore, 2008); H.-P. Breuer and F. Petruccione, The Theory of Open Quantum Systems (Oxford University Press, New York, 2007); C. W. Gardiner and P. Zoller, Quantum Noise (Springer, Berlin, 2004).

[13] A. Arenas, A. Diaz-Guilera, J. Kurths, Y. Moreno, and C. Zhou, Phys. Rep. 469, 93 (2008).

[14] K. R. Brown, C. Ospelkaus, Y. Colombe, A. C. Wilson, D. Leibfried, and D. J. Wineland, Nature (London) 471, 196 (2011); M. Harlander, R. Lechner, M. Brownnutt, R. Blatt, and W. Hansel, ibid. 471, 200 (2011); M. R. Hush, W. Li, S. Genway, I. Lesanovsky, and A. D. Armour, Phys. Rev. A 91, 061401(R) (2015).

[15] R. Vasile, F. Galve, and R. Zambrini, Phys. Rev. A 89, 022109 (2014).

[16] A. W. Chin, A. Rivas, S. F. Huelga, and M. B. Plenio, J. Math. Phys. 51, 092109 (2010); R. Martinazzo, B. Vacchini, K. H. Hughes, and I. Burghardt, J. Chem. Phys. 134, 011101 (2011).

[17] J. Nokkala, F. Galve, R. Zambrini, S. Maniscalco, and J. Piilo, Sci. Rep. 6, 26861 (2016).

[18] Á. Rivas, S. F. Huelga, and M. B. Plenio, Rep. Prog. Phys. 77, 094001 (2014); I. de Vega and D. Alonso, arXiv:1511.06994 [Rev. Mod. Phys. (to be published)].

[19] I. de Vega, Phys. Rev. A 90, 043806 (2014). 
[20] F. Galve, A. Mandarino, M. G. A. Paris, C. Benedetti, and R. Zambrini, arXiv:1606.03390, and references therein.

[21] E. Kajari, A. Wolf, E. Lutz, and G. Morigi, Phys. Rev. A 85, 042318 (2012).

[22] A. Wolf, G. De Chiara, E. Kajari, E. Lutz, and G. Morigi, Europhys. Lett. 95, 60008 (2011); T. Fogarty, E. Kajari, B. G. Taketani, A. Wolf, T. Busch, and G. Morigi, Phys. Rev. A 87, 050304 (2013).

[23] T. Zell, F. Queisser, and R. Klesse, Phys. Rev. Lett. 102, 160501 (2009).

[24] L. A. Correa, A. A. Valido, and D. Alonso, Phys. Rev. A 86, 012110 (2012)

[25] V. Ameri, M. Eghbali-Arani, A. Mari, A. Farace, F. Kheirandish, V. Giovannetti, and R. Fazio, Phys. Rev. A 91, 012301 (2015).

[26] P. Hanggi and G.-L. Ingold, Chaos 15, 026105 (2005).

[27] R. J. Rubin, Phys. Rev. 131, 964 (1963).

[28] H.-P. Breuer, E.-M. Laine, J. Piilo, and B. Vacchini, Rev. Mod. Phys. 88, 021002 (2016).

[29] J. F. Kimble, in Fundamental Systems in Quantum Optics, edited by J. Dalibard, J.-M. Raimond, and J. Zinn-Justin, Proceedings of the Les Houches Summer School of Theoretical Physics, LIII, 1990 (Elsevier, Amsterdam, 1992), pp. 545-674.
[30] J. W. Strutt (Lord Rayleigh), The Theory of Sound, 2nd ed. (Dover, New York, 1945), Vol. 1.

[31] J. K. Knowles, Struct. Control Health Monit. 13, 324 (2006); T. Balendra, C. W. Tat, and S. L. Lee, Earthquake Eng. Struct. Dyn. 10, 735 (1982).

[32] T. K. Caughey, J. Appl. Mech. 27, 269 (1960); T. K. Caughey and M. E. J. O'Kelly, ibid. 32, 583 (1965).

[33] Here "classical" is the adopted terminology used in vibration physics, not to be confused with the absence of quantumness.

[34] F. Galve, G. L. Giorgi, and R. Zambrini, Phys. Rev. A 81, 062117 (2010).

[35] F. Galve and R. Zambrini, Int. J. Quantum Inf. 12, 1560022 (2014).

[36] G. L. Giorgi, F. Plastina, G. Francica, and R. Zambrini, Phys. Rev. A 88, 042115 (2013).

[37] Primed frequencies include the Lamb shifted one due to coupling with the chain.

[38] B. G. Taketani, T. Fogarty, E. Kajari, T. Busch, and G. Morigi, Phys. Rev. A 90, 012312 (2014).

[39] R. Horodecki, P. Horodecki, M. Horodecki, and K. Horodecki, Rev. Mod. Phys. 81, 865 (2009). 